Itinéraires Itinéraires

Littérature, textes, cultures

2020-3 | 2021

Le rap, une poésie de performances

\title{
Entre médiation, tiraillement et éclatement, la crise identitaire de l'écrivain-narrateur d'autobiographie intellectuelle
}

Les cas de : Roland Barthes par Roland Barthes, de Roland Barthes ; Écrire en pays dominé, de Patrick Chamoiseau ; La vie sans fards, de Maryse Condé ; Le Scribe et son ombre, d'Abdelkébir Khatibi ; Ma grandmère bantoue et mes ancêtres les Gaulois, d'Henri Lopes

Between Mediation, Tension and Explosion, The Identity Crisis of the WriterNarrator of Intellectual Autobiography

\section{Stéphane Ledien}

\section{OpenEdition}

Édition électronique

URL : https://journals.openedition.org/itineraires/8859

DOI : $10.4000 /$ itineraires.8859

ISSN : 2427-920X

Éditeur

Pléiade

Référence électronique

Stéphane Ledien, «Entre médiation, tiraillement et éclatement, la crise identitaire de l'écrivainnarrateur d'autobiographie intellectuelle », Itinéraires [En ligne], 2020-3 | 2021, mis en ligne le 09 décembre 2021, consulté le 12 décembre 2021. URL : http://journals.openedition.org/itineraires/8859 ; DOI : https://doi.org/10.4000/itineraires.8859

Ce document a été généré automatiquement le 12 décembre 2021.

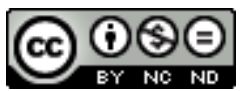

Itinéraires est mis à disposition selon les termes de la licence Creative Commons Attribution - Pas d'Utilisation Commerciale - Pas de Modification 4.0 International. 


\section{Entre médiation, tiraillement et éclatement, la crise identitaire de l'écrivain-narrateur d'autobiographie intellectuelle}

Les cas de : Roland Barthes par Roland Barthes, de Roland Barthes ; Écrire en pays dominé, de Patrick Chamoiseau; La vie sans fards, de Maryse Condé ; Le Scribe et son ombre, d'Abdelkébir Khatibi ; Ma grandmère bantoue et mes ancêtres les Gaulois, d'Henri Lopes

Between Mediation, Tension and Explosion, The Identity Crisis of the WriterNarrator of Intellectual Autobiography

Stéphane Ledien

Lorsqu'il affirmait : "qui parle (dans le récit) n'est pas qui écrit (dans la vie) et qui écrit n'est pas qui est », Roland Barthes (1966: 20) s'inspirait du psychanalyste Jacques Lacan, qui avait lui-même formulé l'interrogation suivante : «le sujet dont je parle quand je parle est-il le même que celui qui parle?» On le sait, pour Barthes, narrateur et personnages sont des «êtres de papier», l'auteur «matériel»-la personne de l'écrivain - d'un récit ne pouvant alors «se confondre en rien avec le narrateur de ce récit » (19). Rejetant par là même "l'indéfectible puissance » du « mythe littéraire » qui veut " voir dans le narrateur et les personnages des personnes réelles, "vivantes" », le critique et sémiologue apportait en fait avec cette assertion un éclairage nouveau à la catégorie philosophico-linguistique de la notion d'auteur, entité jusque-là conçue, pour reprendre les termes de la chercheuse et professeure Élizabeth Molkou, «comme une présence subjective originelle et donc responsable de son discours» (2003: 151). Transformé en abstraction échappatoire, le concept d'« auteur » désignerait désormais un entre-deux fuyant, une « tangence - entre l'intérieur et l'extérieur de la fiction - où s'aventure l'être écrivant et parlant quand il se présente à la fois comme l'agent et l'objet réel du discours" (Quaghebeur 2008: 337). Mais la résolution barthienne fait émerger une indétermination : qu'advient-il si le texte en présence appartient au genre 
de l'autobiographie, des Mémoires, du journal intime ou de l'autofiction? Toute progressiste qu'elle soit, la tentative de Barthes d'établir «la primauté du "je" non subjectif et textuel comme créateur/producteur du discours» (Molkou 2003: 151), problématise la position du narrateur et sa relation avec la «personne physique » de l'auteur quand celles-ci sont aussi intimement et concrètement liées que dans ce que le spécialiste Philippe Lejeune appelle « un récit rétrospectif en prose qu'une personne réelle fait de sa propre existence, lorsqu'elle met l'accent sur sa vie individuelle [et] [...] l'histoire de sa personnalité » (Lejeune 1975 : 14). Avec le " pacte autobiographique » se trouve affirmée et assumée la fusion entre auteur, narrateur et personnage, superposition identitaire qui, confrontée au postulat de Barthes, pourrait a priori mener à une incongruence : d'autobiographique, ce pacte en deviendrait « oxymoronique ${ }^{1}$ ». Reste que la pratique décryptée par Lejeune et la distinction opérée par Barthes se situent dans deux registres de recherche différents, le genre littéraire d'un côté, le signe linguistique de l'autre. Ce qui interpelle, pour ne pas dire bouscule, le lecteur dans l'affirmation du sémiologue, c'est sa réduction extrême de l'instance narrative au langage, sa suppression du référent au réel qu'est la personne de l'auteur. Plus étonnant encore, l'auteur de $S / Z$ et du Degré zéro de l'écriture s'est lui-même pris au « jeu » de l'autobiographie en 1975 avec Roland Barthes par Roland Barthes, un contenu dont il déclarait d'emblée, dans un péritexte reproduisant son écriture manuscrite (donc le signe d'une sorte d'extension charnelle de lui-même), qu'il devait «être considéré comme dit par un personnage de roman ». Un propos qui rejoignait alors ce qu'il avait déclaré quatre ans plus tôt dans la revue Tel Quel, à savoir que "toute biographie est un roman qui n'ose pas dire son nom » (Barthes 1971 : 89). Contradiction ludique - un " pacte qui sert à rompre le pacte ", avance le chercheur Mounir Laouyen (2000) ? Revendication de l'antiréférentialité de toute démarche d'autoportrait? Ou, simplement, volonté de prouver la dissolution de "l'entité auteur» dans le texte cette « scripturation » qui, par le fait même du langage et de la rétrospection, raconte un autre «soi » que la personne physique à l'origine du récit et impliquée dans les faits rapportés ? Sans doute peut-on répondre : « les trois à la fois ».

Quand, dans le fragment «La coïncidence» de Roland Barthes par Roland Barthes, le théoricien se demande à lui-même : «Ne sais-je pas que, dans le champ du sujet, il n'y a pas de référent ?", il répond aussitôt: "Le fait (biographique, textuel) s'abolit dans le signifiant, parce qu'il coïncide immédiatement avec lui » (Barthes 1995 : 61). Avec une sorte de réaffirmation de l'analyse foucauldienne de l'énoncé « je parle » et un rejet du « discours comme communication d'une vérité de la part d'un sujet qui en serait le titulaire et le garant » (Pino Estivill 2014), Barthes statue que «le sujet n'est qu'un effet de langage ». Problème : que deviennent alors le « je » et le « moi » et tous leurs avatars (nous verrons qu'ils sont nombreux, éparpillés, protéiformes) investis corps et âmeparce que mêlés à une dimension réaliste et historique-dans le récit autobiographique intellectuel ? Pour reprendre ce que soulève, non sans une certaine ironie, l'enseignant-chercheur et essayiste René Pommier: "si c'est vraiment le langage qui parle et non pas l'auteur, alors ce n'est pas Roland Barthes qui dit : "C'est le langage qui parle et non pas l'auteur", c'est le langage. Mais, si c'est le langage qui dit que c'est lui qui parle et non pas l'auteur, peut-on le croire? ? (Pommier s. d.).

3 La question n'est pas tant de "pouvoir croire " que de savoir " qui croire ", surtout à l'aune d'un contenu dont le " pacte initial » doit persuader le lecteur que le but est bien le dévoilement de la vérité intime de son auteur (avec, dans les cas qui nous 
intéressent, une concentration sur l'histoire de ses idées). Le corpus abordé ici fait d'emblée ressortir la difficulté, pour les scripteurs, de s'objectiver, notamment dans un désir de sortir d'eux-mêmes afin d'aller vers « l'autre » (celui que représente le lecteur, mais aussi celui qu'incarne, eu égard au contexte de décolonisation de quatre des cinq œuvres étudiées, l'Afrique), constat non pas d'échec mais d'instabilité qui participe à la construction fuyante de l'image de soi. Reniés à titre de figures souveraines, les auteurs/narrateurs réapparaissent ainsi dans le texte en tant que personnages de fiction à travers la figuration du langage ${ }^{3}$ : en se désignant par les pronoms «je", «moi », mais aussi «nous» et même parfois «tu», «vous», «il» ou «elle», les autobiographes intellectuels en présence ne cessent de faire état de «l'inconsistance de [leur] moi et des mirages de [leur] imaginaire» (Pino Estivill 2014), exprimant « une voix renouvelée qui, loin d'être assignable à une identité stable et rassurante, disperse l'existence à travers l'écriture ${ }^{4}$ ( (ibid.).

Dans un premier temps, ce à quoi donne spontanément forme ce langage est un corps palpable, quasi pulsionnel, totalisant et paradoxalement fragmentaire. De la chair du langage au langage de la chair, véritable matérialisation de tropismes et de lieux communs de la sensualité et de l'exotisme, les corps suaves des êtres de ces récits se projettent aussi dans le regard de l'autre, une conscience formelle, charnelle, renforcée par la réflexivité du texte et l'importance thématique de la photographie et de la ressemblance physique.

5 Très vite pourtant, un « je » dialectique se confronte avec un «moi » politique. Il est frappant d'observer alors comment ce «je » et ce «moi » se trouvent tiraillés entre leurs implications et leurs concessions, leurs origines et leur finalité, leur passé et leur futur dans un présent difficilement conciliable avec la rétrospection et un avenir existentiel incertain.

6 Éclatés, dispersés, déchirés, le "je » et le "moi » qui parlent dans ces récits, image projetée de celui qui écrit, elle-même duplication déformée, esthétisée, de l'image de celui qui est, cherchent une troisième voie, un «tiers-espace». Cette identité, ce caractère triple ou ternaire, dépassement d'une dualité initiale mais aussi de cette multiplicité dispersive jusque-là source de souffrances, pourrait bien apparaître comme la résolution de la crise identitaire du narrateur-autobiographe intellectuel.

\section{Émergence des corps et fragments d'un discours désireux : de la chair du langage au langage de la chair}

7 Indéniablement, les autobiographies à l'étude font ressortir l'importance du corps de celui qui les écrit. Parce que le scripteur (pour reprendre le terme utilisé par Khatibi) ne dispose pas de la faculté de se voir lui-même, sauf à se projeter dans un miroir mais il ne contemplerait alors qu'un reflet -, cette matérialisation physique s'effectue toujours par un rapport synecdochique de partie pour le tout: la "main qui trace " chez Roland Barthes ${ }^{5}$ devient ainsi l'image derrière laquelle l'ensemble du texte suivra; celles de Khatibi, le support incontournable d'un «art de s'expliquer » («14 janvier 1983, chirologie", Le Scribe et son ombre $\left.{ }^{6}: 82\right)$, et le "ventre» de Maryse Condé (abandonné, avec sa propriétaire, par Jean Dominique - La vie sans fards ${ }^{7}: 23$ - ou que l'écrivaine elle-même "traînai[t] au collège ", 92), le vecteur fertile d'une destinée soumise à toutes les indécisions et les imprévus. Pour celui qui écrit, observer un fragment de ce qu'il est permet de prospecter une représentation plus juste de lui- 
même et d'extrapoler à partir de là des qualités ou des conditions d'existence plus globales. Les mains comme la tête préfigurent un comportement, un rapport au monde. Mais ces organes isolés peuvent aussi, au contraire, traduire un hiatus entre le raisonné et le ressenti - ce fameux aspect "émotif » dont parle Barthes dans le fragment «Le corps pluriel » (RB: 63). Lorsqu'Henri Lopes confie : « je baissai la tête mais bouillonnai en mon for intérieur " (Ma grand-mère bantoue et mes ancêtres les Gaulois $\left.{ }^{8}: 33\right)$ à propos de la leçon que sa grand-mère lui fit un jour, il laisse mine de rien échapper un aspect majeur de la conscience corporelle - au sein de laquelle s'exprime une tension entre la chair et l'esprit - de l'autobiographe intellectuel. Comme Barthes déclarant que son corps ne lui existe qu'à lui-même que sous la forme " de la migraine et de la sensualité " (RB : 63) ou Khatibi rêvant d'une femme « avec seulement le sexe nu, ouvert » (LS: 82), la présence au monde de l'écrivain-narrateur se fonde sur une réappropriation des sens par l'intermédiaire de ses propres organes ou, comme dans l'exemple khatibien, par ceux d'un tiers, fantasmés ou non.

Ce que l'esprit tait, le corps le dit, et ce que l'esprit confie volontiers à propos du corps fait réagir la chair, même rétrospectivement. Maryse Condé convoque par exemple les passions déchaînées et la voracité charnelle sans fin, ou presque, dont elle a été l'objet : son amour pour Jacques V., fils naturel de François Duvalier, ou ce week-end qu'elle raconte avoir passé à Lagos avec Kwame («nous nous enfermâmes [...] et nous fîmes l'amour quarante-huit heures d'affilée ", $L V: 293-294)$, répondent à tous « ces corps en rut » qu'elle observe régulièrement elle-même (236). Romans - à l'exception de celui de Barthes - de l'africanité et d'un exotisme exacerbé, les autobiographies en présence instaurent et se réapproprient les lieux communs de la sensualité : par exemple, la danse ou le rythme, que la doxa rattache à l'authenticité des gens de couleur, mais que Maryse Condé avoue à maintes reprises ne pas maîtriser du tout; cette «rumba congolaise » dont Lopes se refuse à faire un automatisme lorsqu'il s'agit de fredonner un air ( $M G: 15)$ ou cette évocation, toujours chez Lopes, de la possibilité de « danser d'autres pas que ceux du rythme du tam-tam du village natal» (19). Autres topoï de la sensualité, images - tropes - d'une suavité soumise à une remémoration sensible (et parfois ironique) : la prédominance des fruits, exotiques ou juteux, illustration de sensations parfois déployée avec une fonction quasi métonymique, le fruit désignant le lieu ou l'origine: «Je suis né dans une ville en forme d'orange. [...] Ses maîtres, pour mieux en presser le jus, l'avaient coupée en deux» (MG: 59). Avec Lopes, les indépendances africaines deviennent même un fruit "à cueillir» (MG:23, quand ce fruit est « encore vert ", 34, quand il est enfin mûr). Chez Chamoiseau, le " paysage des champs de cannes » agrippe l'auteur avec une persistance de «sucre fermenté » (Écrire en pays dominé ${ }^{9}: 49$ ), tandis que Maryse Condé émaille son autobiographie d'évocations culinaires et d'expressions du ravissement ressenti sur certains marchés africains ou à la table d'hôtes généreux : ce "repas fort simple » qui «fut un délice » chez Keita Fodéba (LV: 133), ou la "délicieuse nourriture ghanéenne et nigériane " (184). Chamoiseau va plus loin en faisant coïncider beauté du monde créole environnant et écriture au présent :

J'écris parfois sur la terrasse où des colibris boivent à mon eau sucrée [...] Les [...] mots me déportent [...] dans le brasillement de ces pluies que j'adore, dans les couleurs mourantes des bougainvillées, dans l'entrelacs de ces plantes-amies que j'arrose chaque jour, ou dans le vol géométrique des colibris. (EP : 135)

L'auteur-narrateur exprime sa présence au monde de façon sensitive, ancrage qui lui sert de repère tout au long du récit où s'entremêlent souffrance ancestrale et 
contemplation mirifique : "J'émerge sur la terrasse. Les colibris sont là » (EP: 179). Derrière cette "poéticité » du désir tropical (aux déclinaisons volontiers florales chez Lopes ${ }^{10}$ ) s'exprime la tentation du tropisme en général, au-delà même du contexte africain ou caribéen: la photo d'un palmier devient chez Barthes l'allégorie de l'écriture d'un ailleurs, un « arbre-lettre » qui reste « le plus beau » et possède «l'effet majeur ", celui de "la retombée» (RB: 46-47). Qu'elle soit reliée à une perception directe - les saveurs explorées dans «J'aime, je n'aime pas " (RB : 107), l'admiration de Condé pour « la beauté des [Ivoiriennes], [...] leur manière de se vêtir et de se couvrir de

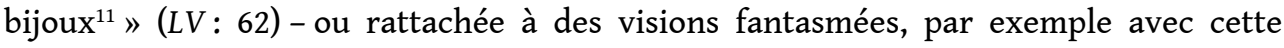
« image du corps ondoyant d'une femme ${ }^{12}$ » (LS: 77), c'est cette même sensualité qui permet d'individualiser le corps de chacun. Un plaisir, écrit encore Barthes, qui «s'oppose à ce que le corps se constitue en lieu étranger » (RB:63). Mais ce mouvement s'avère extensionnel. Comme l'énonce Khatibi de son côté, « décrire un corps » et ses implications sensorielles et érotiques, c'est l'«intersection entre le langage et les quatre éléments " (LS: 77). Au fil de l'écriture autobiographique, le texte s'incarne en corps dont la matérialité s'avère palpable, ouverte. Initié en tant que projection parcellaire, cet organisme de chair dépasse ses attributs sensoriels pour devenir une entité plus globale : corps « couleur» (un mot qui hante sans cesse l'autobiographie de Condé et vient nuancer les discours de Lopes), corps-paysage, corps-pays voire continent (Maryse Condé ne dit-elle pas que l'Afrique «la dénude»?), corps-ethnie (dans $E P$, les corps des esclaves dans la cale d'un négrier renvoient à la totalité originelle de l'actuelle communauté noire hors d'Afrique), et finalement, corpstropiques.

Mais c'est aussi dans le regard de l'autre que ce corps finit par être projeté. L'image photographique s'immisce ainsi comme médium d'une transcendance possible ou, à tout le moins, d'une ressemblance à laquelle se rattache l'écrivain-narrateur pour pouvoir exister: Barthes ouvre son récit sur des «images» sources d'un "plaisir de fascination » (RB:7) et Condé évoque régulièrement des "instantanés de [ses] défunts parents, de [ses] frères, sœurs et [elle] à tous âges » ( $L V: 259)$, symbolisations concrètes et non fugitives (du moins jusqu'à la perte du porte-documents qui les renferme) de sa généalogie. Le texte, impression de signes sur le papier, peut à quelques occasions servir de substitut viable. Quand ses photographies sont perdues, Condé matérialise par exemple l'une d'entre elles par le biais de l'écriture et de la citation intertextuelle ${ }^{13}$. Mais l'instabilité de la retranscription met en péril cette incarnation. Espace de rapprochements physionomistes (Condé ne voit-elle pas l'image de Jean Dominique se répercuter dans celle de son fils Denis ?), le support photographique et son pendant imaginaire et langagier offrent au scripteur qui les contemple la sécurité de sa propre figuration. Tout se passe comme si l'image photographique, par sa captation, demeurait garante de l'intégrité du corps en mouvement/changement. Tandis que Barthes émet une préférence pour les images de sa jeunesse («le temps du récit (de l’imagerie) finit avec la jeunesse du sujet», $R B: 8$ ), Chamoiseau confie que "les photos anciennes [1]'émeuvent plus que les autres ", lui qui se réinvente aussi en "vieux guerrier » tout au long de son récit : "Le vieilli du papier ", écrit-il, « les rides d'un autre temps, les vêtements oubliés, les endroits aujourd'hui transformés ou disparus projettent d'un coup l'éclaboussure des sensations" (EP:38). Cependant, pour l'écrivain originaire de la Martinique, la photo qui a capturé les êtres et les regards «n'intègre nullement leur existence ». C'est la limite, ontologique pourrait-on dire, de la fixation photographique. "Machine ", comme l'énonce Khatibi, «à remonter le temps et à fabriquer à la fois du 
passé et de la mémoire virtuelle», la photographie projette en fait «l'objet dans un faisceau d'images apparemment fixes"; mais cette fixité n'est que "pure illusion, car c'est celui ou celle qui regarde qui la justifie en y déposant la signification qui lui convient" (LS: 123). Tout le danger vient, à l'instar du jeu de miroir évoqué par Khatibi, du risque d'être « un reflet perdu dans le regard de l'autre » (125). Qu'il s'opère à l'appui d'un cliché photographique ou par l'entremise d'une figure de style appropriée (comparaison, métaphore, etc.), le positionnement de l'image de soi par rapport à la vision d'autrui mène au leurre de l'imitation. En sollicitant une « sociabilité cosmopolite ", pour reprendre les mots ultimes de Khatibi, les auteurs-narrateurs ne sont-ils pas condamnés à singer, donc à imiter pour la tourner aussi en dérision, leur propre représentation? La question n'aurait pas tant de sens si deux des auteurs ne jouaient pas brièvement avec les vocables simiesques. "Comme vous, je descends du chimpanzé » lâche d'emblée Lopes à titre de comparaison-généralisation, tandis que Barthes, rapportant un fait historique à propos du gentilhomme Acosta, Juif portugais exilé à Amsterdam et excommunié par les rabbins après qu'il a adhéré à la Synagogue, disserte sur le « vol de langage » dans « Singe parmi les singes » $(R B: 146)$.

Favorable à l'émergence d'un corps envisagé dans ses parties propices à l'appréhension sensible, pour ne pas dire sensuelle, du monde avant d'être soumis à un mouvement extensionnel, le langage des textes en présence met aussi en lumière une aliénation active $\mathrm{du}$ sujet à une image-photographie, souvenir visuel, constat d'une ressemblance physique - qui ne peut totalement servir à un processus d'identification. À travers des récits marqués dans leur chair se crée une crise entre le «je » de chaque auteur-narrateur, et son « moi » instance de l'image et du social.

\section{Un « je » dialectique et un « moi » politique récessif, tiraillé entre histoire et vérité}

11 Ce qui frappe d'emblée à la lecture des œuvres concernées, c'est le positionnement politique de leur sujet dans l'histoire : si Lopes, dont le lecteur connaît le cheminement diplomatique, dévoile cette visée dès le sous-titre de son autobiographie (le mot "discours ", bien que minimisé - perverti, quelque part - par l'adjectif "simples", renvoie à une dimension profondément oratoire), Maryse Condé met en place un processus de persuasion basé sur la primauté du pathos (alternance permanente de l'apitoiement et de l'émerveillement, de l'enchantement et de la désillusion, etc.). À travers les choix relationnels de l'auteure-narratrice se dessine la poursuite d'une légitimation: "Je n'étais pas encore suffisamment "politisée", sans doute ", écrit-elle avec des guillemets de distanciation ( $L V:$ 89). Mais même dans l'effort de remémoration, cette complétude se trouve toujours minorée ou remise à plus tard: « Je n'avais pas vraiment d'avis ", confie-t-elle (250), à un stade avancé du récit, à propos de la situation troublée du Ghana. Attirant de grandes figures du pouvoir africain de l'époque post-coloniale, la narratrice donne l'impression d'exercer malgré elle cette force centripète : "Seul le président feignit de s'intéresser à moi. Sékou Touré était encore plus beau de près. [...] L'adulation dont on l'entourait était palpable » (LV : 91). À tout instant, le récit condéien oscille entre engagement et désengagement ou plutôt : il construit la conviction d'un engagement dans le désengagement - et inversement. Voir à cet égard l'exemple, encore, de Sékou Touré se faisant acclamer par la foule au volant de sa Mercedes : «Apparemment, j'étais la seule à trouver navrant le contraste entre 
cet homme tout-puissant et les pauvres hères faméliques et haillonneux, ses sujets, qui l'applaudissaient» (85). L'engagement qu'on retrouve chez Chamoiseau semble en revanche plus brouillé, a priori soluble dans la poésie et l'intertextualité : dans sa "Sentimenthèque » se côtoient de nombreux auteurs politisés et parfois en lutte contre un régime d'exclusion ou totalitaire: Soljenitsyne, V.S. Naipaul (grand détracteur de l'impérialisme américain et du nationalisme dans le tiers-monde), Breyten Breytenbach (écrivain anti-Apartheid), Guillevic (poète français communiste), etc. C'est que, comme le soulève Barthes, lui-même prompt à évoquer les «t textes révolutionnaires » (RB: 76), le marxisme (115) et «Trotsky, Luxemburg, Bakounine, Mao, Bordiga, etc.» (138), « le texte politique" constitue un "espace obstinément polysémique, le lieu privilégié d'une interprétation perpétuelle » (130). En bref : l'objectivité se trouve court-circuitée par l'affect et la dispersion imaginaire. Ce fait est particulièrement observable chez Lopes, dont le discours politique se nourrit des incertitudes du langage: Lopes joue avec les contenus implicites, les présupposés, les sous-entendus et même les stéréotypes (clichés argumentaires de l'Afrique corrompue ou du génocide nazi). À plusieurs occasions, l'auteur-narrateur confirme, légitime puis révoque des savoirs narratifs déjà constitués, en particulier dans le texte " Débat avec les masses ", au sein duquel se creuse un fossé entre l'époque où le Lopes d'à peine trente ans s'« imaginai[t] tout savoir » (MG : 23) et celle de la remémoration où « le temps a fait son œuvre » (28). Vérité et rétrospection ne font pas bon ménage: «Peut-être ne suis-je [...] qu'un dangereux menteur ", ironise Lopes (111). " Mais un menteur de haut vol, car il s'agit [...] de mentir juste, de "mentir-vrai" » (Ibid.). Dans le récit-discours lopésien truffé d'assertions déguisées, d'euphémismes, d'atténuations et de prétéritions - par exemple cette éloquente fausse interrogation: "Est-il nécessaire d'ajouter un commentaire à cette donnée? » (45) -, le monde et son histoire forment un chiasme, et le pour et le contre s'inversent dans une sorte de dialectique pascalienne. Défi et rétractation se frôlent avec un sens de la ruse rhétorique; l'une des grandes habiletés de $M G$ relève de la liaison du "dire» et du "faire», comme si verbes et actes concrets s'avéraient inconciliables. En témoignent de nombreuses traces de non-fiabilité, entre concession et réfutation, du narrateur: emploi fréquent du conditionnel ou d'un système hypothétique, compléments circonstanciels de manière exprimant une forte subjectivité ("à vrai dire ", "sans doute») ou locutions adverbiales de type «à la limite ». On retrouve une défaillance de logique mémorielle similaire dans l'ouvrage de Condé : «Ma guérison coïncidant avec le début de mes relations avec de nouvelles connaissances, je ne sais pas si celles-ci n'en furent pas la cause » (LV: 98); «Pour des raisons que j'ai oubliées... » (241), « Je crois me souvenir... » (244), « Je crois que ce fut là ma première tentation d'écrire » (247) ; «Je ne me rappelle plus comment j'ai quitté le Ghana » (303). De nombreux modalisateurs conditionnent le récit condéien et sa vérité instable. Comme Lopes tournant sa jeunesse en dérision, Condé remet sans cesse en doute, au présent, les certitudes de son passé, à l'aide de prolepses ironiques ou destinées à prévenir toute objection: "Je ne pouvais pas m'imaginer qu'un jour je reviendrais à ma nationalité française et que je remercierais le ciel de n'avoir, à tort ou à raison, rempli aucun document ce jour-là » $(69) ;$ « Si l'on m'avait prédit que quelques années plus tard, j'épouserais un Anglais et que je finirais par chérir son pays, j'aurais pris cela pour une blague de mauvais goût» (261). En racontant les événements politiques et sociaux qui permettent de se raconter lui-même, l'autobiographe intellectuel se trouve dans une position irréconciliable avec la vérité. L'être de la littérature, disait Roland Barthes, n'est pas dans son message mais dans son langage, 
soit dans un système de signes dont le "sens est suspendu", problématique, introduisant une " déception ", une " déprise » à l'égard du sens et la volonté de vérité qui s'ensuit ${ }^{14}$. La dislocation identitaire, " opposition silencieuse » et " présence close ", d'EP se nourrit par exemple d'un discours constitué d'autres écritures militantes, combatives ou libératrices ; entre unicité et éparpillement, universalité et éclatement, l'existence littéraire du «je » chamoisien est assujettie à une "amplitude ouverte». Dans $L V$ s'exprime une volonté de contrôler à la fois le discours externe et l'ethos de l'auteure-narratrice, tandis que chez Lopes s'observe un déplacement du collectif vers le sujet individuel, posture à contre-courant mais au nom, ici aussi, d'une forme d'universalité pour le bien commun. Individu versus universalité, histoire personnelle contre (et dans) l'histoire collective, tiraillement entre le dire et le faire, mais aussi entre les origines et la finalité : retrouver une Afrique-«Afrique, où es-tu?» questionne Maryse Condé (145) -, oui, mais laquelle? Ou faire face à cette «totalité » qu'évoque Barthes au final? Un conflit - une désunion pourrait-on dire-s'observe aussi entre l'implication et les concessions, entre passé - l'autobiographe « arrache des fragments errants » et les « voit se disperser dans l'air du temps tel un jeu de souvenirs, réels ou fictifs " $(L S: 121)$-, présent et futur ${ }^{15}:$ l'autobiographie telle que la pratiquent les cinq auteurs se révèle source de tensions et de contradictions étrangement complémentaires. Exemple frappant: quand elle se trouve en Afrique, Maryse Condé revendique son origine antillaise; mais face à une "domination" française ou antillaise, elle réaffirme son "africanité ». Tout comme Barthes dans le fragment «Le livre du Moi " énonce que «ce livre n'est pas le livre de ses idées " (RB: 110), les ouvrages du corpus ne sont pas ceux des idées de leurs auteurs, mais plutôt ceux d'une fracture à la fois scripturale et mentale - en bref, d'une récession : c'est «le livre de [leurs] résistances à [leurs] propres idées, [...] un livre récessif (qui recule, mais aussi, peut-être, qui prend du recul)» (ibid.). Ici se rejoignent rapport troublé entre fiction et histoire d'une part, et entre fiction et écriture de soi d'autre part. Ces « romans » (mots qu'emploient volontiers Condé et Barthes) sont aussi à percevoir en tant que tels surtout si on les voit comme des histoires du moi ; un « moi » homme/femme et artiste qui se dévoile, mais dont l'aspect autobiographique est mis sur le même plan que le projet historique. Un tel réalisme subjectif semble correspondre à une exigence de vérité, parce que, comme l'a écrit un théoricien, "pour qu'un récit soit vrai sur le monde extérieur, il faut que la vérité soit dite aussi sur l'auteur de ce récit lui-même ${ }^{16} »$. La vérité sur soi viendrait ainsi compléter la vérité sur l'histoire. Sauf que l'exercice autobiographique représente l'affirmation d'un moi ambigu qui, paradoxalement, peut aussi s'exprimer autrement qu'à la première personne.

Les récits en présence s'imposent en effet comme le lieu d'une "dialectique des déictiques ». Au-delà du «moi » d'Henri Lopes qui, sous l'emprise d'un paternalisme épidictique de bon aloi, se transmue en un " nous " rassembleur ${ }^{17}$, l'objectivation de soi conduit par exemple Barthes à alterner narration autodiégétique ("Quand je jouais aux barres, au Luxembourg...», RB : 55) et narration hétérodiégétique («Enfant, il n'aimait pas tellement les films de Charlot... ", 58) pour parler de lui-même, quitte à se désigner parfois par ses initiales («R. B. semble toujours vouloir limiter la politique »,57) ou à se dédoubler, comme dans le fragment «Sa voix » (processus de dépossession/attribution à un tiers qui n'est que lui-même) : "(Il ne s'agit de la voix de personne. - Mais si ! précisément : il s'agit, il s'agit toujours de la voix de quelqu'un.) Je cherche peu à peu à rendre sa voix » (68). Même dissociation chez Khatibi, dont la voix, dans un dialogue sur "l'hostilité amoureuse", se répartit entre «le discutant» et «le narrateur» (LS: 
92-99). La charge émotionnelle de la reconstitution par le langage trouble le jeu identitaire. "L'imaginaire", écrit Barthes (99), "vient à pas de loup, patinant en douceur sur un passé simple, un pronom, un souvenir, bref tout ce qui peut se rassembler sous la devise même du Miroir et de son Image : Moi, je ». L'écartèlement et l'incertitude forgent le récit-discours de l'autobiographe certes intellectuel, mais aussi émotif. Chez Condé, Lopes et Barthes, le pronom indéfini « on » vient même prendre la relève lorsqu'il s'agit d'énoncer, ou de tenter d'énoncer, une vérité générale : "On n'aurait pas besoin de dire ce mot » $(R B: 104)$, « On aura remarqué... [...] On peut à cet égard comprendre... » (MG: 92), « On imagine toujours que le viol... » (LV:182). Vérité (avec un grand V) et subjectivisme se toisent, s'affrontent. Définitivement affecté par une crise des déictiques ${ }^{18}$, le «je» narrant, parfois remodelé en «nous» ou dépersonnalisé en « on », s'égare en «tu »/« toi »/« vous » (le lecteur et l'Afrique, ou la finitude de l'ouvrage en cours). Son «ici» devient «ailleurs», son passé remémoré s'incarne, par son acte d'énonciation, au présent ou au conditionnel, son " maintenant " renvoie à "hier " voire à un hypothétique "demain », eux-mêmes susceptibles, dans le regard de l'autre, de se transformer en «la veille» ou «le lendemain ».

Déchirées et mises en tension, les instances narratives concernées sont finalement tout autant «multifragmentaires» que duales. Seule solution - peut-être - d'équilibre: la découverte d'un "tiers-espace», siège "triple» d'une identité qui ne les fera plus pencher ni d'un côté, ni de l'autre.

\section{Une autre voie : derrière le multiple et par-delà le clivage, le « triple»}

14 S'ils se dispersent, comme nous l'avons vu, en plus d'une dualité qui les fait osciller entre, en bref, une existence de chair et une condition littéraire, et entre une identité de personne et un statut narratif, les «je » et les «moi » protéiformes de ces récits cette «tendance multipolaire » à laquelle se réfère très tôt Khabiti dans son ouvrage (LS: 23) - n'en explorent pas moins une troisième voie que tous parviennent peu ou prou à atteindre. Qu'on décide de l'appeler tiers-espace ${ }^{19}$ ou non, cette voie médiante se trouve tracée, délinéée, désignée, en filigrane des textes. Pour Khatibi, adepte d'une "fluidité identitaire " (LS: 23 et 117), il pourrait s'agir de ce qu'il nomme «l'Inter ", c'est-à-dire «le lieu neutre où tout se passe, où rien ne se passe » (LS : 117). Serait-ce là le champ de tous les possibles, de l'absolu ou, au contraire, du néant qui, à l'instar du «monstre de la totalité » dont parle Barthes $(R B: 156)$ pourrait tout engloutir? «Tout dépend », précise l'auteur marocain, « de la perspective où l'on se place ». Considérant qu'il s'agit, pour lui, du dernier ouvrage écrit de son vivant, l'idée d'un point de nonretour n'échappe pas au lecteur. Pourtant, l'horizon d'attente positif crée cette possibilité d'une véritable "réincarnation». Vu sous l'angle de la transmutation, le projet de chaque auteur-narrateur prend le chemin de l'adoption d'un axe tiers, une façon d'être qui ne serait plus ni « multi », ni binaire, mais triple ou ternaire. C'est chez Lopes que cette condition apparaît la plus flagrante, paradoxalement dès le début de son autobiographie, dans le texte "Mes trois identités ». " Trois identités, trois cordes d'une même guitare ", conclut Lopes ( $M G: 19)$, faisant converger tout ce qu'il est, parle et écrit, vers un principe d'harmonie totale. À l'unisson de cette "valse » qu'il sifflote parfois (15), le Congolais truffe ses discours de progressions aux indéniables effets 
d'amplitude, mais aussi de phrases au rythme ternaire, dont le plus bel exemple reste cette séquence extraite de "Métis »: "Allez savoir pourquoi, le noir et le blanc ne donnèrent pas du gris, mais du marron ${ }^{20} »(59)$. Chez Barthes, l'espace tiers se devine plus qu'il ne se lit, le sémiologue pointant d'abord du doigt "Le second degré et les autres ». "J'écris : ceci est le premier degré du langage. Puis, j'écris ce que j'écris : c'en est le second degré » (RB:67). Mais si, comme il le précise quelques paragraphes plus loin, "tout discours est pris dans le jeu des degrés» (68), alors la lecture du texte barthien ou son éclosion sur papier, au choix, pourrait très bien s'appréhender comme un "troisième degré ". Trois s'affiche même comme un chiffre de résolution dans le récit de Maryse Condé ("N’empêche! En trois jours, Bankole régla mon problème", $L V: 192$ ), et comme un principe d'identification chez Chamoiseau («moi-Indiens, moiChinois, moi-Syro-Libanais ", EP: 129). C'est aussi une "règle de trois » que Barthes élabore dans le même fragment en détaillant « les instances habituelles de l'expression, de la lecture et de l'écoute », premier «triple » inclus dans un autre, celui qu'il précise aussitôt entre parenthèses: "vérité", "réalité", "sincérité" ». Simultanéité de la personne-auteur, du narrateur et de ses projections fictives? Ou, autrement, coexistence du "lisible », du "scriptible » et (de l') «au-delà », comme pourrait le signifier certain fragment au titre orné de ces trois mots-clés $(R B: 108)$ ?

Si quelque chose se dessine, ou, comme nous l'écrivions plus haut, se trace de luimême, c'est peut-être bien cette sorte de «tiercéité»-pas au sens freudien ou psychanalytique ${ }^{21}$ mais tel qu'envisagé dans la sémiotique de Charles Sanders Peirce ${ }^{22}$ (il avait formulé là, à travers une étude de la substance et de l'être, l'hypothèse de l'existence des trois catégories fondamentales de l'être, parmi lesquelles la Tiercéité) qu'on croit voir surgir dans le texte de Lopes, dans la «mise en signes » de Barthes et, dans une moindre mesure, dans les récits de Condé, de Chamoiseau et de Khatibi. L'écriture autobiographique revêt ainsi les traits d'une médiation vers un espace luimême médian, chaque auteur-narrateur mêlant et mettant à l'œuvre, pour reprendre les termes associés à Peirce, «la pensée, le langage, la représentation », mais aussi la possibilité d'une "communication sociale» et d'un lien avec «la vie intellectuelle». Dépassant le lancinement binaire du "tam-tam» (mot en deux syllabes) ou des griots que convoquent tant de fois Lopes ${ }^{23}$ et Condé2 ${ }^{24}$, «l'Écrire » autobiographique - avec un grand $\mathrm{E}$, référence à Chamoiseau - ouvert « à tous les vents » (MG:113) adopte en fin de compte un schéma, mouvement triple qui lui permet de résoudre l'énigme de sa propre quête («inachevée» dit Lopes, ibid.). Et cela, qu'elle soit le dépassement d'une «absence» ou du «reste» (RB: 156) ou une réinvention, "ré-imagination» de l'Afrique ; cette Afrique qui «n'accepterait jamais » l'auteur-narrateur tel qu'il est ( $L V$ : 144) mais qui, « enfin domptée se métamorphoserait et se coulerait, soumise, dans les replis de [1']imagination » (334).

Matière charnelle et sensible, le texte autobiographique intellectuel en souffrance transcrit une crise à la fois d'identité et de désignation. À l'issue d'une fragmentation et d'une dialectique précédant la découverte d'un «tiers-espace » salvateur, une nouvelle vérité éclate: celle qui correspond à une reconstruction, pour ne pas dire reconfiguration, de la personne - dans son entièreté - dissimulée derrière chacun de ces récits hybrides. Barthes a beau distinguer le « je » de la personne-auteur de celui du narrateur et des personnages, l'autobiographie post-moderne n'hésite pas à bousculer ce repère de la nouvelle critique en brouillant - et même en les écrasant - l'écrire, le parler et l'être du signataire du récit/discours. Peut-être aussi parce que, comme l'a 
écrit le linguiste et sémiologue Georges Mounin, «en réalité, il y a toujours des rapports entre l'homme et l'auteur, rapports divers, complexes, à des niveaux variables selon les auteurs et les œuvres ». " Tout le problème », dit Mounin, « consiste à mettre en relation, de l'auteur à l'œuvre, seulement les faits qui ont une valeur explicative réelle, c'est-à-dire une pertinence du point de vue du fonctionnement de l'œuvre » (Mounin 1978 : 178).

\section{BIBLIOGRAPHIE}

Barthes, Roland, [1964] 1991, Essais critiques, Paris, Seuil.

Barthes, Roland, 1966, «Introduction à l'analyse structurale des récits », Communications, vol. 8, $\mathrm{n}^{\circ} 1$, « Recherches sémiologiques : l'analyse structurale du récit », p. 1-27.

Barthes, Roland, 1971, Tel Quel, nº 47, Paris, Seuil.

Barthes, Roland, 2002, CEuvres complètes, tome III : Livres, textes, entretiens, 1968-1971, Paris, Seuil. Boillet, Étienne, 2016, « Le déni de la fiction dans la poétique narrative de Giorgio Bassani », Fabula / Les colloques, L'Histoire en fictions. La Seconde Guerre mondiale dans le néoréalisme italien, [En ligne], http://www.fabula.org/colloques/document3099.php, consulté le 12 juillet 2021.

Cuillerai, Marie, 2010, «Le Tiers-espace : une pensée de l'émancipation ? ", Acta fabula, vol. 11, $\mathrm{n}^{\circ}$ 1, « Autour de l'œuvre d'Homi K. Bhabha », [En ligne], http://www.fabula.org/revue/ document5451.php, consulté le 12 juillet 2021.

Everaert-Desmedt, Nicole, 2011, « La sémiotique de Peirce », Signo, Site Internet de théories sémiotiques, [En ligne], http://www.signosemio.com/peirce/semiotique.asp, consulté le 12 juillet 2021.

Jaccomard, Hélène, 1993, Lecteur et lecture dans l'autobiographie française contemporaine : Violette Leduc, Françoise d'Eaubonne, Serge Doubrovsky, Marguerite Yourcenar, Genève, Droz.

Laouyen, Mounir, 2000, «L'autofiction : une réception problématique », Fabula, Colloque en ligne, Frontières de la fiction, [En ligne], https://www.fabula.org/anciens_colloques/frontieres/ 208.php, consulté le 12 juillet 2021.

Lejeune, Philippe, 1975, Le Pacte autobiographique, Paris, Seuil.

Lyotard, Jean-François, 1978, Discours, figure, Paris, Klincksieck.

Mengue, Philippe, 2003, «Deleuze et la question de la vérité en littérature », E-rea, Revue électronique d'études sur le monde anglophone, vol. 1, nº 2, [En ligne], https://doi.org/10.4000/erea. 371.

Molkou, Élizabeth, 2003, «L'autofiction : un genre frontière ? ", dans N. Martinière et S. Le Ménahèze (dir.), Écrire la frontière, Limoges, Presses universitaires de Limoges, p. 147-159.

Mounin, Georges, 1978, La Littérature et ses technocraties, Paris, Casterman, coll. « Synthèses contemporaines ». 
Ody, Michel, 2005, « Notes théoriques et cliniques sur la tiercéité », Revue française de psychanalyse, vol. 69, p. 861-868, [En ligne], https://doi.org/10.3917/rfp.693.0861.

Pino Estivill, Ester, 2014, « L'écriture barthésienne contre l'oubli (vue depuis l'Espagne) », r.bRevue Roland Barthes, $\mathrm{n}^{\circ} 1$ - Jeunes chercheurs, juin 2014, [En ligne], http://www.rolandbarthes.org/article_pino.html, consulté le 12 juillet 2021.

Pommier, René, s. d., "Quand un auteur mort-né, Roland Barthes, décrète la mort de l'auteur ", Assez décodé!, Site de René Pommier, [En ligne], http://rene.pommier.free.fr/Auteur.htm, consulté le 12 juillet 2021.

Quaghebeur, Marc (dir.), 2008, Analyse et enseignement des littératures francophones : tentatives, réticences, responsabilités, Bruxelles, PIE Peter Lang, coll. « Documents pour l'histoire des francophonies ».

Van Elslande, Jean-Pierre, 2003, «La mise en scène du discours », Université de Neuchâtel : Département de français moderne, Méthodes et problèmes, [En ligne], http://www.unige.ch/lettres/ framo/enseignements/methodes/srhetorique/rdintegr.html, consulté le 12 juillet 2021.

\section{Corpus}

Barthes, Roland, 1975, Roland Barthes par Roland Barthes, Paris, Seuil. [RB]

Chamoiseau, Patrick, 1997, Écrire en pays dominé, Paris, Gallimard. [EP]

Condé, Maryse, 2012, La Vie sans fards, Paris, Éditions Jean-Claude Lattès. [LV]

Khatibi, Abdelkébir, 2008, Le Scribe et son ombre, Paris, Éditions de la Différence, coll. «Littérature ». [LS]

Lopes, Henri, 2003, Ma grand-mère bantoue et mes ancêtres les Gaulois. Simples discours, Paris, Gallimard, coll. « Continents Noirs ». [MG]

\section{NOTES}

1. L'expression a été proposée par Hélène Jaccomard dans Lecteur et lecture dans l'autobiographie française contemporaine: Violette Leduc, Françoise d'Eaubonne, Serge Doubrovsky, Marguerite Yourcenar (1993).

2. Roland Barthes par Roland Barthes (1975) ; Patrick Chamoiseau, Écrire en pays dominé (1997) ; Maryse Condé, La vie sans fards (2012) ; Abdelkébir Khatibi, Le Scribe et son ombre (2008) ; Henri Lopes, Ma grand-mère bantoue et mes ancêtres les Gaulois. Simples discours (2003). C'est à ces éditions / collections précises que nous nous reporterons. Voir section Corpus en bibliographie.

3. Cette hypothèse renvoie à ce qu'explique Paul de Man dans l'article « Autobiography as De-facement» (1979), propos relayé par Ester Pino Estivill dans «L'écriture barthésienne contre l'oubli (vue depuis l'Espagne)» (2014) : «l'autobiographie est une figure rhétorique du langage - prosopon poien (masque et personne) - à travers laquelle, dans la tragédie classique, on donnait voix aux morts et aux absents. Dans l'autobiographie, cette figure nous confère un langage qui nous permet de nous figurer à nous-mêmes, en même temps qu'il nous dépossède de toute identité assignable. » 
4. Ou comme l'a écrit une fois de plus Barthes: «L'écriture est destructrice de toute voix, de toute origine. L'écriture, c'est ce neutre, ce composite, cet oblique où fuit notre sujet, le noir-et-blanc où vient se perdre toute identité, à commencer par celle même du corps qui écrit » $(2002: 40)$.

5. « ... pour qu'il soit libre de ses signes propres, jamais figuratifs, le texte suivra sans images, sinon celles de la main qui trace» (Barthes 1995: 9). Désormais, nous désignerons l'ouvrage par l'abréviation « $R B »$.

6. Nous désignerons désormais l'ouvrage par l'abréviation « $L S »$.

7. Nous désignerons désormais l'ouvrage par l'abréviation « $L V$ ».

8. Nous désignerons désormais l'ouvrage par l'abréviation « $M G »$.

9. Que nous désignerons désormais sous l'abréviation « $E P$ ».

10. Voir dans $M G$, l'emploi fréquent que l'auteur fait du champ lexical des plantes et de métaphores végétales: il parle d'une "société fleurie» (34), d'un patrimoine de l'humanité à «fertiliser » (40), ou encore de « mains chargées de fleurs » et d'« espèces aux pétales inconnus ", de "fleurs et [...] plantes [qui] ne poussent pas au même mois, à la même saison et dans les mêmes conditions sous tous les climats » (96). Lopes poétise beaucoup sur le cycle de la nature, pas seulement d'ailleurs pour une connexion esthétique au monde créole : également pour mettre l'accent sur un ordre des choses fragile dans un écosystème à protéger et à apprécier. Il y a dans ces évocations un certain pathos rousseauiste avec une idéalisation de la nature.

11. On pourrait aussi citer ces trois passages «impressionnistes » de $L V$, où affleurent lieux communs de la couleur exotique (et de la vastitude africaine) et "métonymisation» de l'Afrique ensoleillée: «Le nez collé au hublot, j'observai en frissonnant l'épais tapis vert sombre de la forêt qui se déroulait sous l'appareil, le rouge sanguinolent de la terre, puis l'océan démesuré, éblouissant » (63); « Je vis que le soleil brillait, que le ciel et la mer étaient bleus, que les amandiers bordant la plage de Boulbinet étaient verts et rouges » (98); « je découvrais l'ivresse du large. [...] Tout cet azur autour de moi m'enivrait » (144).

12. Fantasmé ou anticipé, comme dans cet autre passage de $L S:$ «... je n'ai pu voir la beauté de la célèbre pierre de Jérusalem, ni ses reflets, ni ses réverbérations lorsqu'elle reçoit les changements colorés du ciel » (107).

13. " Je décris dans Le cœur à rire et à pleurer une photographie perdue comme les autres qui est restée gravée dans mon souvenir : "Mes frères et sœurs en rang d'oignons. Mon père, moustachu, vêtu d'un pardessus à revers de fourrure façon pelisse. Ma mère, souriant de toutes ses dents de perle, ses yeux en amande étirés sous son taupé gris." " (LV: 259).

14. Barthes ([1964] 1991), cité et éclairé par Philippe Mengue (2003).

15. Par exemple : tout en évoquant l'histoire ancienne du continent africain, Lopes se situe abondamment dans le genre délibératif, genre de discours de persuasion qui "s'efforce d'amener l'auditoire à prendre une décision qui engage l'avenir " (Van Elslande 2003).

16. La phrase est extraite d'une étude d'Étienne Boillet à propos de l'œuvre romanesque de Giorgio Bassani. "Ces réflexions", écrit Boillet, "portent sur des genres qu'on a tendance à distinguer voire à opposer: le roman réaliste et l'autobiographie. L'histoire littéraire est le plus souvent vue comme un balancement 
entre concentration centripète des écrivains sur leur ego [...] et attention centrifuge au monde extérieur (le réel, la société, l'Histoire) » (Boillet 2016).

17. «En effet, dans les littératures militantes, les auteurs disent plus souvent nous que je. [...] Quand Césaire, Senghor et Damas, pères de la négritude, disaient je, nous savions que c'était nous qu'ils entendaient » (MG : 18).

18. Ou ce que le philosophe Jean-François Lyotard appelle une «diadéictique »: «La diadéictique peut être une sorte de dialectique [...] ; une dialectique, parce qu'il est vrai que le je et le ici s'engendrent ensemble dans le mouvement qui parcourt et qu'il crée ce qu'il parcourt, de même que le sujet et l'objet de la parole naissent aux deux pôles de son mouvement de signifier » (Lyotard $1978: 39$ ).

19. Théorie post-coloniale qu'a formalisée Homi K. Bhabha (chercheur d'ailleurs cité par Maryse Condé, p. 294-295 de LV), dans son ouvrage Les lieux de la culture - Une théorie postcoloniale ([1994] 2007). Bhabha y « développe une réflexion sur l'altérité qui déplace la référence identitaire du sujet [...]. L'identité y devient un phénomène susceptible d'hybridations [...] qui se transportent en des lieux provisoires et fragiles, “interstitiels" "(Cuillerai 2010).

20. À noter qu'il y a en plus ici une progression et une figure de gradation, voire une hyperbole, le troisième terme de la phrase indiquant un dépassement du "conflit » entre les deux premiers.

21. À propos de cette tiercéité-là, lire par exemple : Michel Ody, « Notes théoriques et cliniques sur la tiercéité » (2005).

22. "La tiercéité est la médiation par laquelle un premier et un second sont mis en relation. La tiercéité est le régime de la règle, de la loi ; mais une loi ne se manifeste qu'à travers des faits qui l'appliquent, donc dans la secondéité ; et ces faits eux-mêmes actualisent des qualités, donc de la priméité. [...] La tiercéité est de l'ordre du nécessaire et, par conséquent, de la prédiction. La loi de la pesanteur, par exemple, nous permet de prédire que chaque fois que nous lâcherons une pierre, elle tombera sur le sol. La tiercéité est la catégorie de la pensée, du langage, de la représentation, du processus sémiotique; elle permet la communication sociale; elle correspond à la vie intellectuelle » (Everaert-Desmedt 2011).

23. «Car, prophétise un adage congolais: "Aujourd'hui, tu sculptes le tam-tam dans la solitude, demain il fera danser le pays." » (MG:19).

24. «Paroles musiques où les sons s'interpellent et se répondent » (LV: 136). « Nous avancions péniblement, assourdis par les battements du tamtam et les hurlements des griots... » (190). "Une demi-douzaine de batteurs en casaques et pantalons bouffants rouges frappaient en désordre sur leurs tam-tams... » (233). «Pas un bruit, hormis le halètement feutré de la mer. Je distinguai dans le lointain le battement d'un tamtam... » (236). 


\section{RÉSUMÉS}

Cet article analyse les tensions qui habitent l'écrivain engagé dans un exercice d'autobiographie intellectuelle. L'étude est en particulier appliquée à un corpus postcolonial et plus précisément à un ensemble de textes d'auteurs caractérisés par une certaine hybridation culturelle, que celle-ci ait impliqué un parcours de migration ou pas. En contrepoint de ces œuvres aux dimensions politiques, poétiques et sociologiques, la lecture de Roland Barthes ouvre le champ des considérations linguistiques. En partant donc d'une problématisation à la fois sémiologique et littéraire, l'articulation s'articule en trois temps. Il s'agit d'abord de s'interroger sur la manière dont, pour chacun des textes, le langage autobiographique donne corps au « je » en inscrivant le récit dans une dimension charnelle. L'attention est ensuite portée à l'inscription d'un «je » dialectique dans l'histoire politique de l'Afrique et de ses diasporas, mais aussi dans le monde scriptural et littéraire. Le dernier point aborde la dimension tierce de l'énonciation comme espace de déploiement d'une identité tiraillée. Si, en filigrane, les textes font émerger le concept d'autobiographie intellectuelle comme écriture de résistance des auteurs à leurs propres idées, ils laissent aussi entrevoir la possibilité, pour chacun des auteurs-narrateurs, de surmonter ou de déjouer cette résistance en se raccrochant à ce que certains d'entre eux appellent le «tiersespace».

This article analyzes the tensions that inhabit the writer engaged in an exercise of intellectual autobiography. The study is in particular applied to a postcolonial corpus and more precisely to a set of authors' texts characterized by a certain cultural hybridization, whether this involved a migration route or not. As a counterpoint to these works with political, poetic and sociological dimensions, reading Roland Barthes opens up the field of linguistic considerations. Starting from a problematization that is both semiological and literary, the articulation is articulated in three stages. It is first a question of questioning the way in which, for each of the texts, the autobiographical language gives shape to the "I" by inscribing the story in a carnal dimension. Attention is then paid to the inscription of a dialectical "I" in the political history of Africa and its diasporas, but also in the scriptural and literary world. The last point addresses the third dimension of enunciation as a space for the deployment of a torn identity. If, in the background, the texts bring out the concept of intellectual autobiography as a writing of resistance by authors to their own ideas, they also suggest the possibility, for each of the author-narrators, of overcoming or thwarting this resistance by hanging on what some of them call "third space".

INDEX

Mots-clés : autobiographie, autobiographie intellectuelle, décolonisation, littérature postcoloniale, francophonie, pacte de lecture, pacte autobiographique

Keywords : autobiography, intellectual autobiography, decolonization, postcolonial studies, francophonie, dialogic pact, autobiographical pact

\section{AUTEUR}

\section{STÉPHANE LEDIEN}

Enseignant-chercheur, écrivain de fiction, Université Laval (Québec, Canada) 